\title{
miR-202 Enhances the Anti-Tumor Effect of Cisplatin on Non-Small Cell Lung Cancer by Targeting the Ras/MAPK Pathway
}

\author{
Wei Sun Wei Ping Yitao Tian Wenbin Zou Jiawei Liu Yukun Zu \\ Department of Thoracic Surgery, Tongji Hospital, Tongji Medical College, Huazhong University of \\ Science and Technology, Wuhan, China
}

\author{
Key Words \\ miR-202 $・$ KRas $・$ MAPK $・$ Cisplatin $・ N S C L C$
}

\begin{abstract}
Background/Aims: KRas is usually mutated in non-small cell lung cancer (NSCLC). The mutated KRas gene is a negative prognostic indicator that promotes tumor proliferation, metastasis, and drug resistance in NSCLC, and thus has become a target for cancer therapy. This study is focused on the effects of the microRNA (miR)-202/KRas axis in regulating chemosensitivity in NSCLC. Methods: Quantitative reverse transcriptase real-time PCR analysis was performed to examine the expression of miR-202. MTT (3-(4,5-dimethylthiazol-2-yl)-2,5diphenyltetrazolium bromide) assays were performed to evaluate the sensitivity of cisplatin against NSCLC cells. The miR-202/KRas axis was confirmed by western blot and luciferase reporter assays. Cell apoptosis was measured by flow cytometry. KRas expression, MEK1/2 and ERK1/2 phosphorylation, and activation of caspase- 9 and caspase- 3 were detected by western blot. Results: A significant decrease in miR-202 expression was observed in NSCLC cells both in vivo and in vitro. In addition, miR-202 expression was associated with drug resistance. Recovery of miR-202 expression levels was found to increase the sensitivity of both $\mathrm{NCl}-\mathrm{H} 441$ and A549 NSCLC cells to cisplatin treatment. Mechanically, as the Ras/mitogenactivated protein kinase (MAPK) pathway was aberrantly activated in $\mathrm{NCl}-\mathrm{H} 441$ and A549 NSCLC cells, the overexpression of miR-202 was found to inhibit the Ras/MAPK pathway by targeting the KRas gene. As a result, increased miR-202 expression expanded apoptosis signaling induced by cisplatin in NSCLC cells. Conclusion: The miR-202/KRas axis controlled the chemosensitivity of NSCLC by mediating the Ras/MAPK pathway. Thus, the combination of platinum-based drugs with miR-202 may represent a novel strategy to enhance the antitumor effect against NSCLC.
\end{abstract}




\section{Cellular Physiology Cell Physiol Biochem 2018;51:2160-2171 and Biochemistry Published \begin{tabular}{l|l} 
DOI: 10.1159/000495835 & $\begin{array}{l}\text { (c) } 2018 \text { The Author(s). Published by S. Karger AG, Basel } \\
\text { www.karger.com/cpb }\end{array}$
\end{tabular} \\ Sun et al.: miR-202 Enhances Cisplatin on NSCLC}

\section{Introduction}

Non-small cell lung cancer (NSCLC) is a type of cancer that is one of the most common and lethal malignant tumors worldwide [1,2]. For patients diagnosed with early-stage NSCLC, surgery is the most effective treatment strategy. Unfortunately, a large proportion of NSCLC patients are diagnosed at an advanced stage with unresectable tumors. For these patients, systemic chemotherapy is an irreplaceable treatment $[3,4]$. One challenge, however, is that many NSCLC cases exhibit poor response to chemotherapy [5, 6]. New strategies to increase the response of NSCLC cells to chemotherapy are urgently needed.

Ras protein family members are GTPase proteins that initiate the mitogen-activated protein kinase (MAPK) and phosphatydilinositol-3-kinase (PI3K) signaling pathways to regulate cell proliferation, survival, and chemosensitivity [7, 8]. In human cells, the Ras family includes the HRas, NRas, and KRas proteins, which are highly homologous. In NSCLC, there is a high incidence of KRas mutations. It has been reported that oncogenic KRas mutations are present in approximately $15-30 \%$ of all NSCLC cases. Furthermore, KRas mutations are a negative prognostic indicator because they promote tumor proliferation, metastasis, and drug resistance in NSCLC [9-12]. Thus, KRas has become a target for cancer therapy.

MicroRNAs (miRNAs) are a class of small, non-coding, and evolutionarily conserved RNAs. miRNAs bind to the $3^{\prime}$ untranslated region (3'-UTR) of targeted mRNAs, thereby inducing translational repression. Because miRNAs regulate approximately $60 \%$ of all human mRNAs, nearly every cellular process is modulated by miRNAs [13-15]. Due to the importance of miRNAs in regulating cellular processes, miRNA dysregulation is a frequent occurrence in cancers. It has been reported that cancer cells, including NSCLC cells, change their miRNA expression profile to promote cell survival, proliferation, and metastasis, and to develop drug resistance [16-19]. Studies have demonstrated that correcting the altered miRNA profile is a potential strategy for cancer therapy. In the present study, we investigated the effects of miR-202, which is a potential tumor suppressor for cisplatin treatment in NSCLC.

\section{Materials and Methods}

\section{NSCLC cells and tissues}

The NSCLC cell lines NCI-H441 and A549 were both purchased from the American Type Culture Collection (Manassas, VA). They were maintained in Dulbecco's modified Eagle's medium (Gibco, Gaithersburg, MD) supplemented with $10 \%$ fetal bovine serum (Gibco) at $37^{\circ} \mathrm{C}$ in a humidified $5 \% \mathrm{CO}_{2}$ incubator. For the detection of miR-202 in vivo, a total of 25 primary NSCLC tumor tissues and their corresponding paracancerous, non-tumor tissues were obtained from NSCLC patients who underwent tumor resection at Tongji Hospital, Tongji Medical College, Huazhong University of Science and Technology from October 2014 to January 2017. The use of specimens in this study was approved by the ethics committee of Tongji Hospital, Tongji Medical College, Huazhong University of Science and Technology, and all of the patients provided informed consent.

\section{Quantitative reverse transcriptase real time PCR ( $q R T-P C R$ )}

Total RNA was extracted from the NSCLC cell lines and patients' tissues using TRIzol ${ }^{\circledR}$ (Thermo Fisher Scientific, Inc., Waltham, MA). For analysis of miR-202 expression, total RNA was reverse transcribed using stem-loop RT primers from the PrimeScript RT reagent kit according to the manufacturer's protocol (Takara Bio, Inc., Otsu, Japan). The miR-202 RT primer (Ribobio, Guangzhou, China) had the following sequence: $5^{\prime}$-CTCAACTGGTGTCGTGGAGTCGGCAATTCAGTTGAGCAAAGAAG- ${ }^{\prime}$. qRTPCR was performed in triplicate using SYBR Premix Ex Taq II (Takara Bio, Inc.) on an ABI PRISM 7900 Sequence Detection system (Applied Biosystems, Foster City, CA). The forward amplification primer was 5'-ACACTCCAGCTGGGTTCCTATGCATATACT- 3 ', and the reverse primer was 5'-TGGTGTCGTGGAGTCG-3'. The relative expression of miR-202 was determined using the $2^{-\Delta \Delta C q}$ analysis method [20], whereby U6 snRNA was used as an internal reference. 


\section{Cellular Physiology Cell Physiol Biochem 2018;51:2160-2171 \begin{tabular}{ll|l} 
and Biochemistry Published online: 6 December 2018 & $\begin{array}{l}\text { C } 2018 \text { The Author(s). Published by S. Karger AG, Basel } \\
\text { www.karger.com/cpb }\end{array}$ \\
\hline
\end{tabular}}

Sun et al.: miR-202 Enhances Cisplatin on NSCLC

\section{Transfection}

For the direct knockdown of KRas, KRas small interfering RNA (siRNA) was purchased from Santa Cruz Biotechnology (Santa Cruz, CA). For enforced expression of miR-202, the miR202 mimic (5'-UUCCUAUGCAUAUACUUCUUUG-3') and its negative control oligonucleotide (NCO; 5'-UGUAUUCACAUUGUCAUUCUCU-3') were purchased from GenePharma Company (Shanghai, China). For transfection, $50 \mathrm{pmol} / \mathrm{ml}$ of miR-202 mimics, NCO, and KRas siRNA were transfected into either NCI-H441 or A549 cells using Lipofectamine 2000 (Invitrogen, Carlsbad, CA) according to the manufacturer's protocols.

\section{Cell viability assay}

A total of $5 \times 10^{3}$ transfected NSCLC cells were seeded in 96-well plates overnight. Then, the cells were treated with different concentrations of cisplatin, oxaliplatin, or carboplatin for 48 h. Cell viability was evaluated by the 3-(4, 5-dimethylthiazol-2-yl)-2, 5-diphenyltetrazolium bromide (MTT) assay [21]. The absorbance in each well was measured at $570 \mathrm{~nm}$ using a microplate reader (Sunrise Microplate Reader, TECAN, Männedorf, Switzerland). In addition, the half maximal inhibitory concentrations ( $\mathrm{IC}_{50}$ ) of cisplatin, oxaliplatin, and carboplatin against both NCI-H441 and A549 cells were all calculated according to the viability curves.

\section{Western blot analysis}

Cells were lysed in RIPA lysis buffer (Cell Signaling Technology, Danvers, MA). The total proteins extracted from the lysed cells were separated by $12.5 \%$ sodium dodecyl sulfate polyacrylamide gel electrophoresis and transferred to a polyvinylidene fluoride membrane (Millipore, Burlington, MA). The membranes were then probed overnight with primary antibodies (including anti-human KRas, GAPDH, MEK1/2, phosphorylated MEK1/2, ERK, phosphorylated ERK1/2, cytochrome c, apoptosis inducing factor [AIF], Smac/DIABLO, caspase-9, and caspase-3) purchased from Cell Signaling Technology. Subsequently, the membranes were incubated with the appropriate horseradish peroxidase-conjugated secondary antibodies for $2 \mathrm{~h}$ followed by detection with an enhanced chemiluminescence detection kit (Pierce, Rockford, IL). In addition, before the detection of cytochrome c, AIF, and Smac/DIABLO in the cytoplasm of NSCLC cells, cellular mitochondria was removed using a Mitochondria/Cytosol Fraction Kit (BioVision, Milpitas, CA) according to the manufacturer's instructions.

\section{Apoptosis analysis}

After treatment with miR-202 (50 pmol/ml) and cisplatin $(2 \mu \mathrm{M})$ for $48 \mathrm{~h}$, cells were stained with annexin V/propidium iodide (Sigma-Aldrich, St. Louis, MO) for $15 \mathrm{~min}$ at room temperature according to the manufacturer's instructions. Cell apoptosis was analyzed using flow cytometry (Becton Dickinson, Franklin Lakes, NJ).

\section{Luciferase reporter assay}

The KRas 3'-UTR was cloned downstream of the firefly luciferase gene using the pGL3 Luciferase Reporter Vector Selector (Promega, Madison, WI) according to the manufacturer's instructions. To perform the luciferase reporter assay, NSCLC cells were incubated in 48-well plates overnight. The cells were then cotransfected with the pGL3 vectors carrying the KRas 3'-UTR, Renilla luciferase pRL-TK vectors (Promega), and the miR-202 mimics using Lipofectamine 2000. Forty-eight hours after transfection, the cells were collected and lysed. Luciferase activity was then measured using the Dual Luciferase Reporter Assay System (Promega). The relative firefly luciferase activity was normalized to Renilla luciferase activity.

Tumor growth in nude mice

A stable A549 cell line overexpressing miR-202 (LV-miR-202-A549) was generated using a lentiviralbased system (Genechem Co., Ltd., Shanghai, China) for use in tumorigenesis assays. Xenograft tumors were generated via the subcutaneous injection of LV-miR-202-A549 or LV-control-A549 (5 × $10^{6}$ cells) into nude mice (BALB/c, nu/nu; 17-22 g in weight). After the xenografts reached $0.5 \mathrm{~cm}$ in diameter, animals were treated with cisplatin i.p. twice a week $(2 \mathrm{mg} / \mathrm{kg})$ until the mice were sacrificed 28 days post-injection. The animal care and experimental protocols were both approved by the Animal Care Committee of Tongji Hospital, Tongji Medical College, Huazhong University of Science and Technology. 


\section{Cellular Physiology Cell Physiol Biochem 2018;51:2160-2171 \begin{tabular}{ll|l} 
and BiOChemistry & $\begin{array}{l}\text { DOI: 10.1159/000495835 } \\
\text { Published online: 6 December } 2018\end{array}$ & $\begin{array}{l}\text { ( } 2018 \text { The Author(s). Published by S. Karger AG, Basel } \\
\text { www.karger.com/cpb }\end{array}$ \\
\hline
\end{tabular}

\section{Statistical analysis}

Data are represented as the mean \pm standard deviation and were obtained from three independent experiments. For the comparison analysis, one-way analysis of variance was used to determine the differences between groups. Statistical analysis was performed using SPSS 15.0 software (SPSS Inc., Chicago, IL). $P<0.05$ was considered to indicate a statistically significant difference.

\section{Results}

miR-202 is downregulated in KRas-mutant NSCLC in vivo and in vitro

To investigate the expression profile of miR-202 in NSCLC, we analyzed the miR-202 expression levels in NSCLC patients' tumor and paracancerous tissues. The in vivo results showed a significant decrease in miR-202 expression in NSCLC tissues compared with noncancerous tissues (Fig. 1A). Next, to evaluate the expression profile of miR-202 in vitro, we chose two NSCLC cell lines, NCI-H441 and A549, that each harbor the KRas mutation [22]. Similar to the NSCLC in vivo results, the two NSCLC cell lines exhibited significant decreases in miR-202 expression compared with non-tumor cells (Fig. 1B). Together, the in vivo and in vitro results indicated that miR-202 is downregulated in NSCLC.

Recovery of miR-202 expression increases the sensitivity of KRas-mutant NSCLC cells to cisplatin treatment

To investigate the association between miR-202 and chemosensitivity in NSCLC, we transfected both NCI-H441 and A549 cells with a miR-202 mimic in order to recover the downregulated cellular levels of miR-202. As shown in Fig. 2A, enforced expression of miR202 increased the cellular levels of miR-202 in both NCI-H441 and A549 cells. Interestingly, we observed that enforced expression of miR-202 significantly increased the sensitivity of NSCLC cells to cisplatin-induced cytotoxicity in vitro (Fig. 2B). These results indicated that the combination of miR-202 and cisplatin may represent a potential strategy for enhancing the effectiveness of chemotherapy in NSCLC.

\section{miR-202 targets}

KRas in NSCLC

To understand how miR-202 enhanced c i s p lat in - ind u c ed cytotoxicity against NSCLC, we searched for potential targets by using the public miRNA prediction databases TargetScan, miRanda, and PicTar. Each of these databases showed that the KRas gene contained a highly conserved sequence paired with miR-202 located in the 3 '-UTR of its mRNA (Fig. 3A). As miR-202 sensitized KRas-mutant NSCLC cells (NCI-H441 and A549), we predicted that miR-202 targets KRas in NSCLC. To

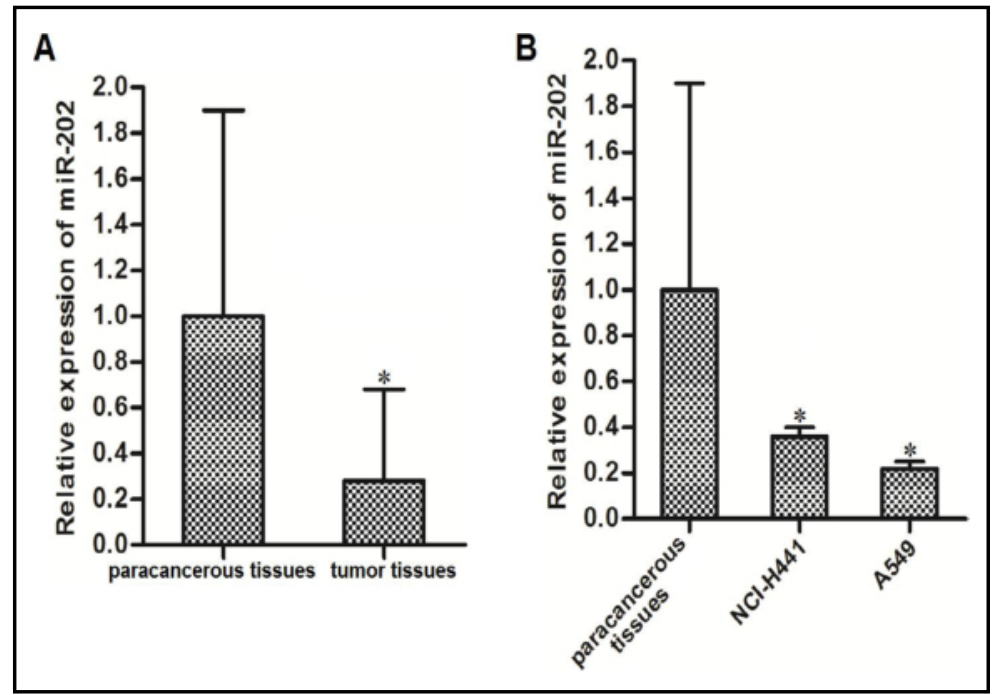

Fig. 1. Expression of miR-202 in NSCLC. A: Patients' cancerous and paracancerous tissues $(n=25)$ were collected. Expression of miR-202 in tumor tissues and the corresponding paracancerous tissues were detected by qRT-PCR analysis. ${ }^{*} \mathrm{P}<0.05$ vs. paracancerous tissues. $\mathrm{B}$ : miR202 expression was downregulated in the NSCLC cell lines NCI-H441 and A549. ${ }^{*} \mathrm{P}<0.05$ vs. paracancerous tissues. 


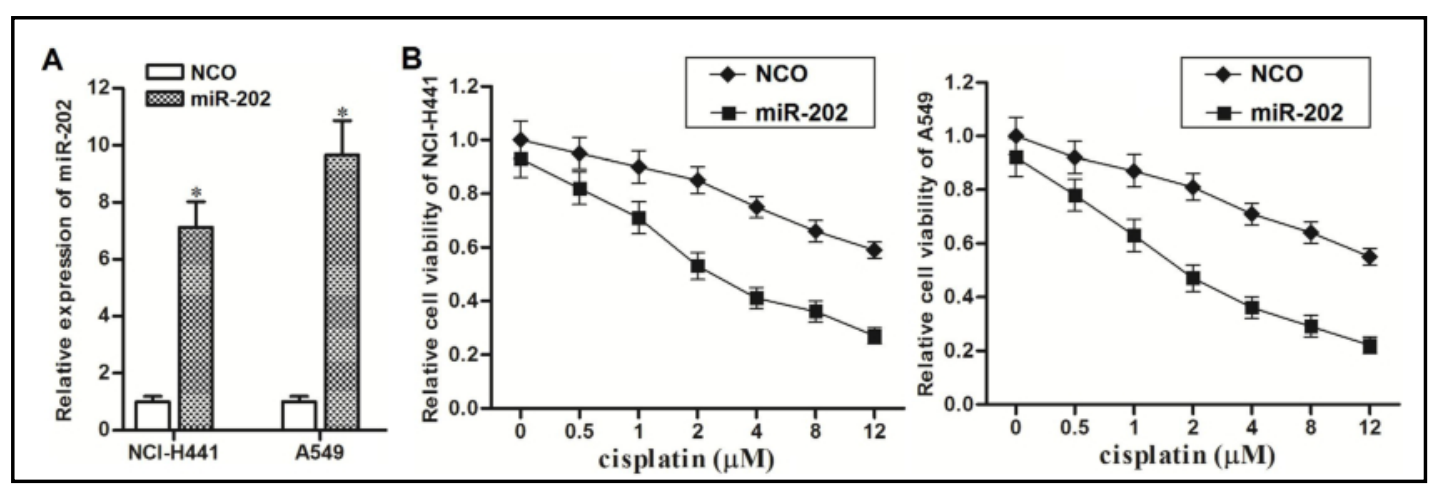

Fig. 2. miR-202 increases the sensitivity of NSCLC cells to cisplatin treatment. A: Transfection efficiency of the miR-202 mimic ( $50 \mathrm{pmol} / \mathrm{ml}$ ) in NCI-H441 and A549 NSCLC cell lines *P<0.05 vs. the NCO group. B: Effects of miR-202 (50 pmol/ml) on cisplatin sensitivity toward NCI-H441 and A549 NSCLC cells.

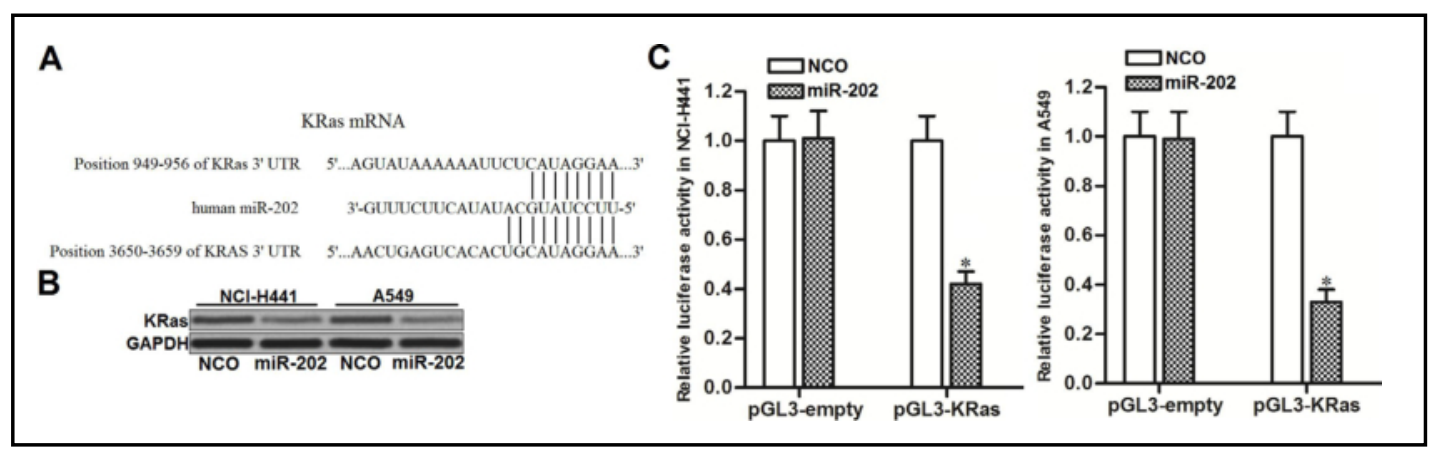

Fig. 3. KRas was the target of miR-202 in NSCLC. A: Sequence of KRas 3'-UTR seed regions paired with miR-202 as was predicted by the public miRNA databases TargetScan, miRanda, and PicTar. B: Western blot analysis was performed to evaluate the inhibitory effect of miR-202 on KRas expression in NCI-H441 and A549 NSCLC cells. C: NCI-H441 and A549 cells were both co-transfected with miR-202 and a pGL3 plasmid contained KRas 3'-UTR or an empty pGL3 plasmid. At $48 \mathrm{~h}$ post-transfection, luciferase activities were measured using the Dual-Luciferase Reporter Assay System according to the manufacturer's instructions. $* \mathrm{P}<0.05$ vs. the NCO group.

confirm this speculation, we detected the KRas protein in both NCI-H441 and A549 cells after they were transfected with the miR-202 mimic. The results of western blot analysis showed that the overexpression of miR-202 dramatically decreased the expression levels of KRas in NCI-H441 and in A549 cells (Fig. 3B). Next, we performed luciferase reporter assays after co-transfection with miR-202 and a pGL3 plasmid containing KRas 3'-UTR or an empty pGL3 plasmid. The results showed that co-transfection with miR-202 decreased the luciferase activities of pGL3-KRas reporters but did not decrease the activity of the empty pGL3 reporters (Fig. 3C). Taken together, we demonstrated that miR-202 targeted KRas in NSCLC.

miR-202 increases the sensitivity of NSCLC cells to cisplatin through suppression of the KRas/MAPK pathway

Next, we studied the role of the miR-202/KRas/MAPK pathway in increasing cisplatin sensitivity in NSCLC cells. As shown in Fig. 4A, miR-202 decreased KRas expression in cisplatin-treated NCI-H441 and A549 NSCLC cells. Furthermore, we found that the overexpression of miR-202 inhibited the phosphorylation of MEK and ERK because the oncogenic KRas is upstream of the MAPK pathway [7, 8]. To emphasize the importance of KRas inhibition in miR-202-promoted cell death in cisplatin-treated NSCLC cells, we performed a KRas loss-of-function assay by transfection with KRas-specific siRNA. We found 


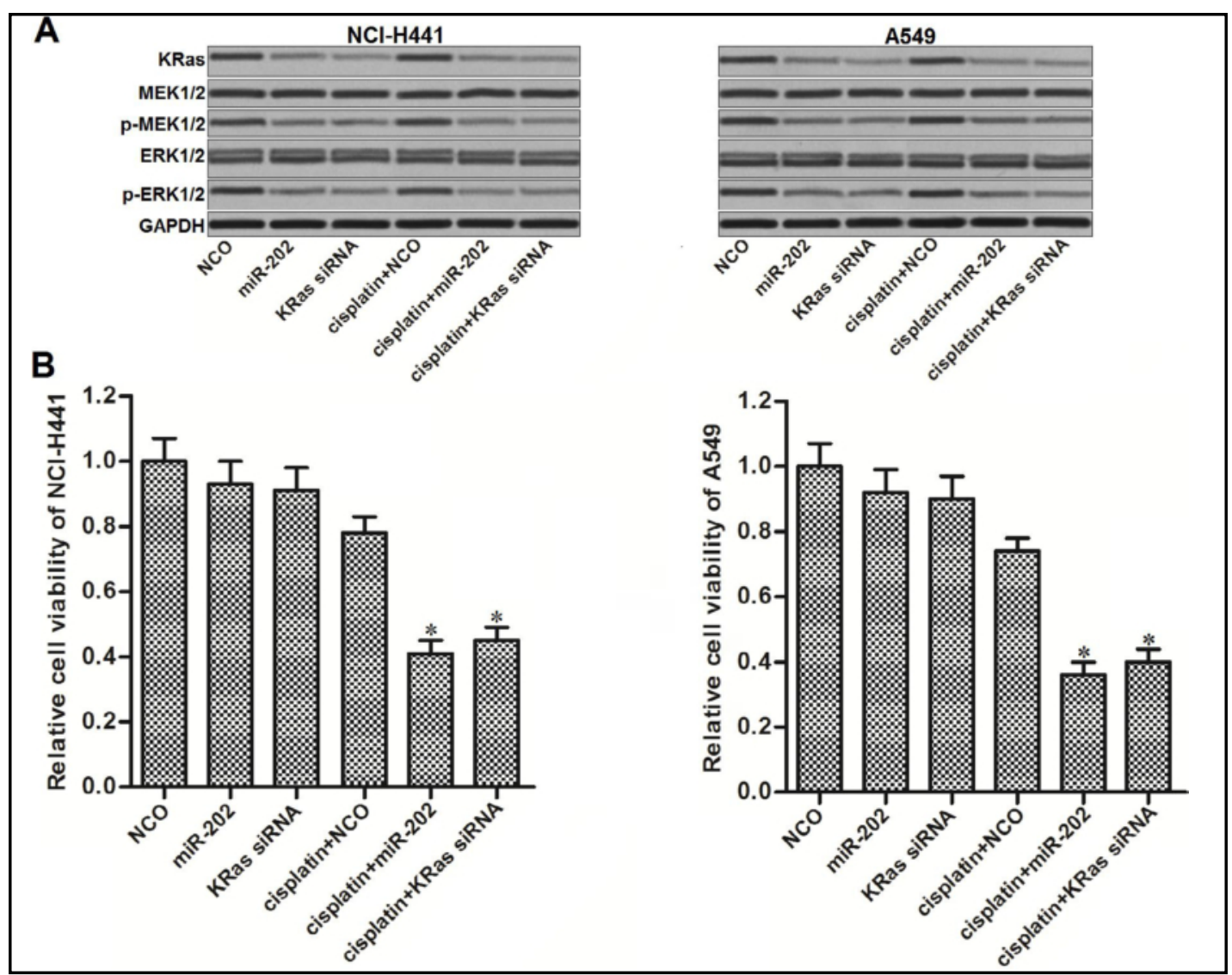

Fig. 4. miR-202 increased the sensitivity of NSCLC cells to cisplatin treatment through suppression of the KRas/MAPK pathway. A: After combination treatment of cisplatin $(2 \mu \mathrm{M})$ and miR-202 $(50 \mathrm{pmol} / \mathrm{ml})$, the expression of KRas and phosphorylation of MEK and ERK were evaluated by western blot analysis. B: After treatment with cisplatin $(2 \mu \mathrm{M})$, miR-202 (50 pmol/ml), and KRas siRNA (50 pmol/ml), cell viability of NCI-H441 and A549 NSCLC cells was detected by MTT assays. ${ }^{*} \mathrm{P}<0.05$ vs. the cisplatin + NCO group.

that the direct knockdown of KRas by using its specific siRNA also enhanced the anti-tumor effect of cisplatin on NSCLC, similar to the combinatory effect with miR-202 (Fig. 4B). Taken together, these results suggested that the miR-202/KRas axis determined the sensitivity of KRas-mutant NSCLC cells to cisplatin treatment. The combination of miR-202 and cisplatin was able to enhance cytotoxicity against NSCLC by suppressing the KRas/MAPK pathway.

miR-202 increases the sensitivity of NSCLC cells to cisplatin-induced apoptosis

Previous studies have demonstrated that the aberrant activation of MAPK pathway induces resistance to apoptosis in cancer cells [23, 24]. Because miR-202 regulates the KRas/MAPK pathway, we decided to investigate the effect of miR-202 on cisplatin-induced apoptosis in NSCLC cells. As shown in Fig. 5A, miR-202 treatment did not induce a significant release of cytochrome $\mathrm{c}$, AIF, or the second mitochondria derived activator of caspase/direct IAP binding protein with low pI (Smac/DIABLO) from the mitochondria into cytoplasm. miR-202 treatment did, however, significantly enhance the effect of cisplatin on the release of these apoptotic inducers. As a result, we found that the combination of cisplatin and miR202 induced significant cleavage of caspase- 9 and caspase-3, which were the main apoptotic markers (Fig. 5B). Overexpression of miR-202 was also shown to enhance cisplatin-induced apoptosis. In fact, the apoptotic rate of NSCLC cells in the cisplatin plus miR-202 treatment group was significantly higher than the cisplatin-only treatment group (Fig. 5C). Taken together, we demonstrated that miR-202 increased the sensitivity of NSCLC cells to cisplatininduced apoptosis by suppressing the KRas/MAPK pathway. 


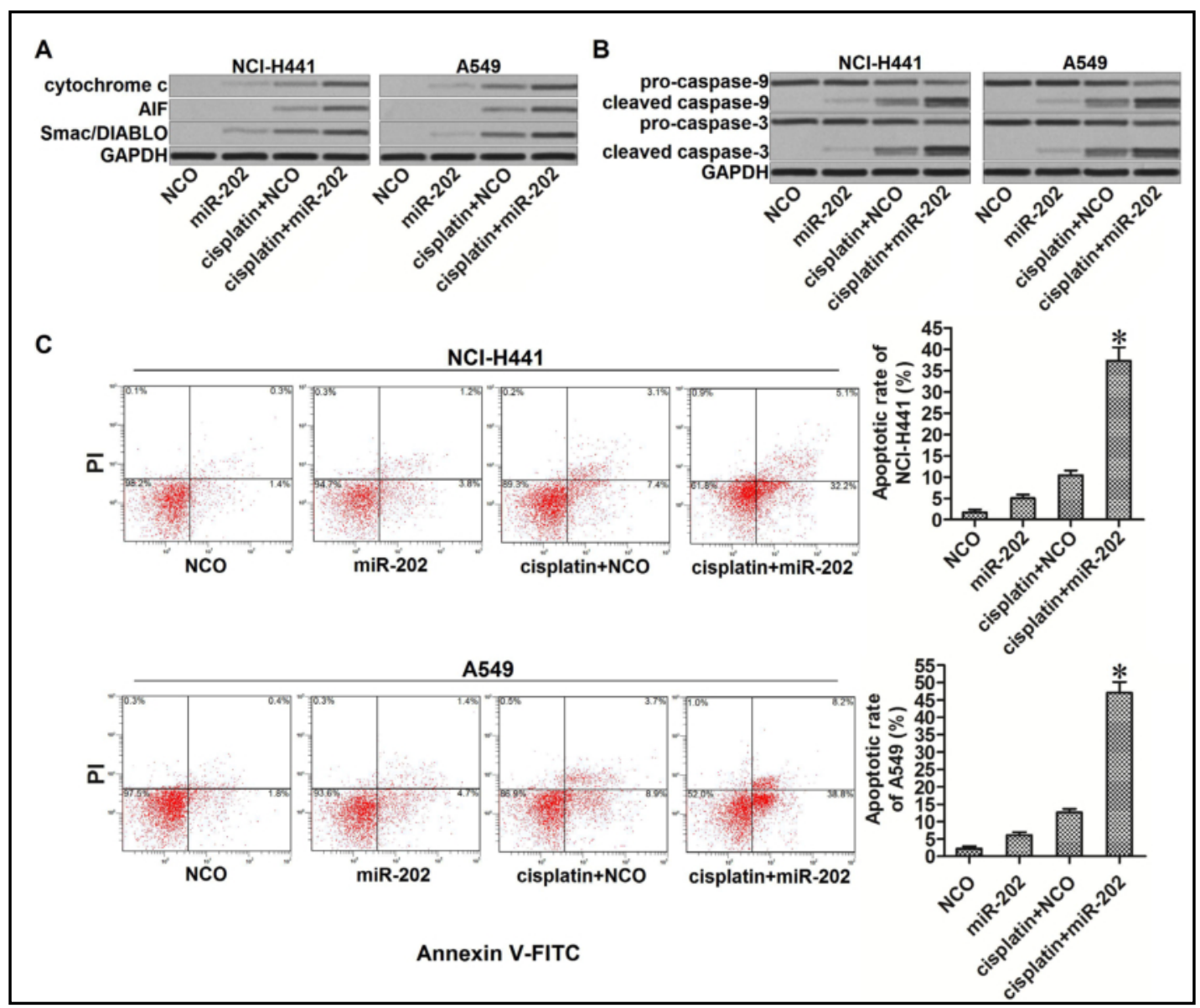

Fig. 5. miR-202 enhanced cisplatin-induced apoptosis by suppressing the KRas/MAPK pathway. A: After treatment with cisplatin $(2 \mu \mathrm{M})$ and miR-202 $(50 \mathrm{pmol} / \mathrm{ml})$, western blot analysis was performed to evaluate the release of cytochrome c, AIF, and Smac/DIABLO from mitochondria into the cytoplasm in NCI-H441 and A549 cells. B: Cleavage of caspase- 9 and caspase-3 in NCI-H441 and A549 cells treated with cisplatin (2 $\mu \mathrm{M})$ and miR-202 (50 pmol/ml). C: Flow cytometry analysis was performed to detect the apoptotic rate of NCI-H441 and A549 cells treated with cisplatin $(2 \mu \mathrm{M})$ and miR-202 $(50 \mathrm{pmol} / \mathrm{ml}) .{ }^{*} \mathrm{P}<0.05$ vs. the cisplatin + NCO group.

Enforced expression of miR-202 enhances the anti-tumor effect of cisplatin on NSCLC in vivo

Next, to investigate the effect of exogenous miR-202 on cisplatin treatment in vivo, we inoculated nude mice with A549 cells transfected with lentivirus carrying miR-202 precursor (LV-miR-202-A549) or with the empty lentivirus. After treating both groups with equal doses of cisplatin, we found that the growth speed of miR-202-overexpressed NSCLC tumors was much slower than that of the tumors transfected with empty lentivirus (Fig. 6A). After sacrificing the mice, tumor tissues were collected and weighed. Tumor tissues from the cisplatin and miR-202 co-treatment group were significantly smaller than those from the cisplatin only group (Fig. 6B). In addition, as miR-202 expression was increased in LV-miR202-transfected tumor tissues (Fig. 6C), the expression level of KRas and phosphorylation of MEK and EKR were inhibited in miR-202-overexpressed NSCLC tumors (Fig. 6D). These results together showed that the recovery of miR-202 inhibited the KRas/MAPK pathway, and thus enhanced the anti-tumor effect of cisplatin on NSCLC in vivo. 
A

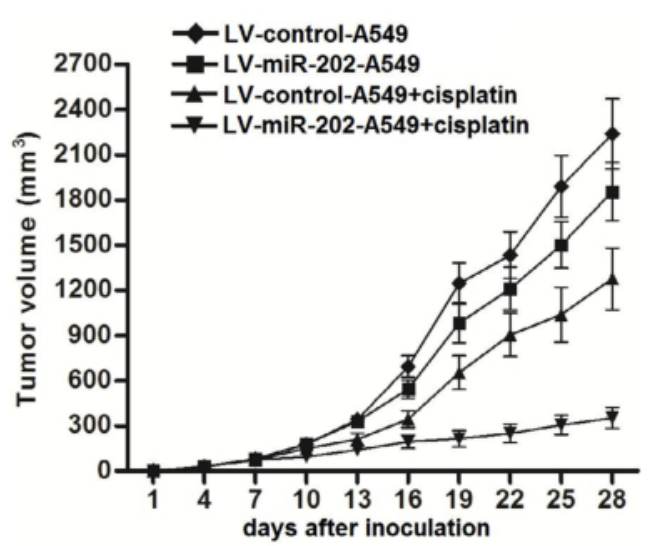

B

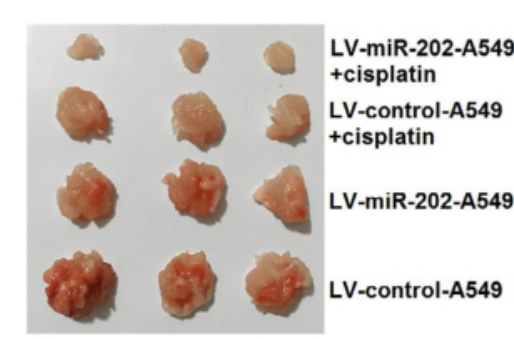

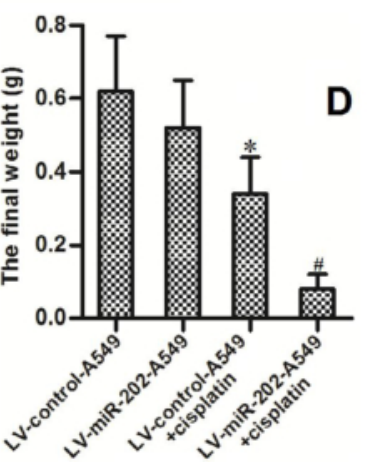

C

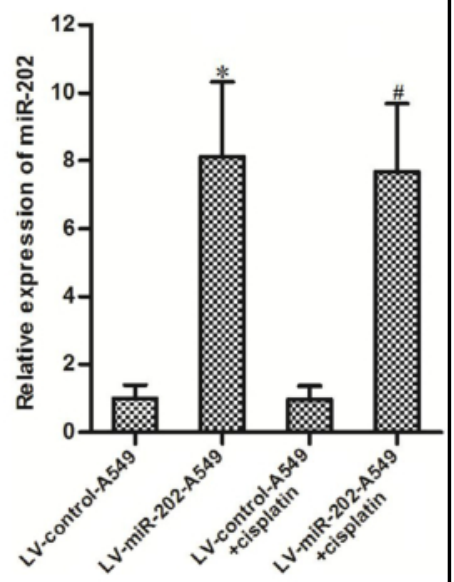

D

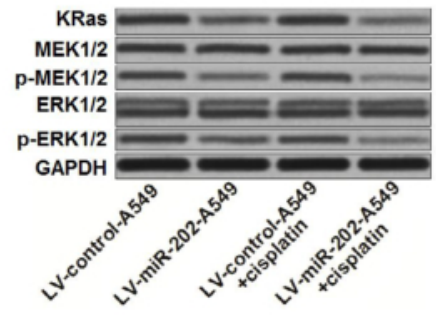

Fig. 6. Overexpression of miR-202 enhanced the anti-tumor effect of cisplatin on NSCLC in vivo. A: After inoculation with LV-miR-202-A549 or LV-control-A549, nude mice were treated with $2 \mathrm{mg} / \mathrm{kg}$ cisplatin twice a week. Tumor volumes were measured every three days until the experimental endpoint was reached (28 days post-injection). B: After sacrificing the mice, tumors were collected and weighed. ${ }^{\mathrm{P}}<0.05$ vs. the LVcontrol-A549 group. ${ }^{\text {P }<0.05}$ vs. the LV-control-A549 + cisplatin group. C: Expression levels of miR-202 by tumor tissue group were detected using qRT-PCR analysis. ${ }^{*} \mathrm{P}<0.05$ vs. the LV-control-A549 group. ${ }^{*} \mathrm{P}<0.05$ vs. the LV-control-A549 + cisplatin group. D: Expression levels of KRas and phosphorylation of MEK and EKR in tumor tissue groups were evaluated by western blot analysis.

Recovery of miR-202 expression increases the sensitivity of NSCLC cells to other platinumbased chemotherapeutic drugs

As described in this study, our results demonstrated that the recovery of miR-202 expression increased the sensitivity of KRas-mutant NSCLC cells to cisplatin treatment. Fig. $7 \mathrm{~A}$ shows that combination treatment with miR-202 decreased the $\mathrm{IC}_{50}$ value for cisplatin on both NCI-H441 and A549 cell lines. To explore whether miR-202 had a broad-spectrum effect on other platinum-based chemotherapeutic drugs or if it was limited to combination with cisplatin, we next investigated the effect of miR-202 on oxaliplatin and carboplatininduced cytotoxicity against NCI-H441 and A549 cells. As shown in Fig. 7B and 7C, enforced expression of miR-202 was found to decrease the $\mathrm{IC}_{50}$ for both oxaliplatin and carboplatintreated NCI-H441 and A549 NSCLC cells. These results indicated that the combination of other platinum-based chemotherapeutic drugs with miR-202 was also a potential strategy to enhance the anti-tumor effect against KRas-mutant NSCLC. 


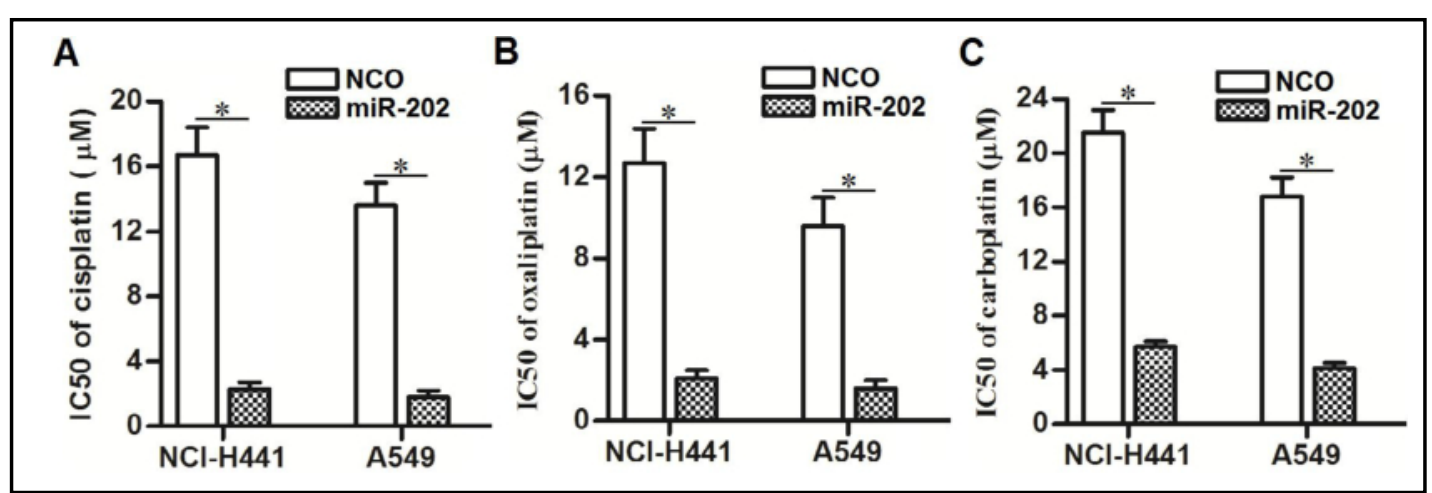

Fig. 7. miR-202 had broad-spectrum effect on multiple platinum-based chemotherapeutic drugs. A: miR$202(50 \mathrm{pmol} / \mathrm{ml})$ significantly decreased the $\mathrm{IC}_{50}$ of cisplatin in NCI-H441 and A549 cell lines. ${ }^{*} \mathrm{P}<0.05$. B: miR-202 (50 pmol/ml) significantly decreased the IC $_{50}$ of oxaliplatin in NCI-H441 and A549 cell lines. ${ }^{*} \mathrm{P}<0.05$. C: miR-202 (50 pmol/ml) significantly decreased the $\mathrm{IC}_{50}$ of carboplatin in NCI-H441 and A549 cell lines. ${ }^{*} \mathrm{P}<0.05$.

\section{Discussion}

Platinum-based chemotherapy is an important and effective strategy for treatment of NSCLC. Among the platinum-based chemotherapeutic drugs, cisplatin is the most commonly used anti-tumor drug for treating advanced NSCLC [25-27]. Usually, in cancer cells, cisplatin induces DNA damage by forming cross-links with DNA, and thus activating the apoptosis signaling pathways $[28,29]$. NSCLC cells, on the other hand, develop drug resistance to cisplatin in many cases $[30,31]$. Our research goal in this study was to enhance the effectiveness of cisplatin treatment on NSCLC cells.

Previous studies have shown that the miRNA expression profile in cancer cells is correlated with cell survival, proliferation, metastasis, and drug resistance. Among these cancer-related miRNAs, miR-202 is reported to function as a tumor suppressor as well as to suppress cell proliferation, tumor progression, and drug resistance in several cancers. Cancers in which miR-202 is reported to have these effects include cervical cancer, hepatocellular carcinoma, colorectal carcinoma, multiple myeloma, and NSCLC [32-36]. Therefore, increasing the expression level of miR-202 in cancer cells may be considered as a novel strategy for cancer treatment.

In this study, we found that the expression profile of miR-202 was changed in NSCLC. We observed that miR-202 was downregulated in both NSCLC tissues and cell lines. These results suggested a potential anti-tumor effect of miR-202 on NSCLC. Next, we artificially increased the cellular level of miR-202 in KRas-mutant NSCLC cells by transfection with a miR-202 mimic. By doing so, we found that the increased expression of miR-202 sensitized these KRas-mutant NSCLC cells to cisplatin-induced cytotoxicity in vitro and in vivo. Thus, we reported that the combination of cisplatin with miR-202 was a novel approach to enhance the treatment effectiveness of KRas-mutant NSCLC cells.

In NSCLC, KRas mutations occur with a high frequency. The oncogenic mutant KRas is responsible for resistance to anti-cancer drug treatments through the constitutive activation of RAS signaling pathways [37, 38]. Downstream of the Ras signaling pathways, the MAPK pathway has been shown to be important for promoting cell proliferation and inhibiting apoptosis in cancer cells. Furthermore, evidence shows that aberrant activation of the MAPK pathway is responsible for cisplatin resistance in NSCLC cells [39-41]. Thus, the KRas/MAPK pathway has been identified as a target for cancer therapy. 


\section{Cellular Physiology Cell Physiol Biochem 2018;51:2160-2171 \begin{tabular}{l|l|l} 
and Biochemistry Published online: 6 December 2018 & $\begin{array}{l}\text { C) 2018 The Author(s). Published by S. Karger AG, Basel } \\
\text { www.karger.com/cpb }\end{array}$ \\
\hline
\end{tabular} \\ Sun et al.: miR-202 Enhances Cisplatin on NSCLC}

In this study, we found that the KRas gene was targeted by miR-202. We also demonstrated that the recovery of miR-202's expression level decreased the mutant KRas in NSCLC cells. Furthermore, our results indicated that overexpression of miR-202 suppressed the aberrant activation of the MAPK pathway through the miR-202/KRas axis. As a result, we found that the sensitivity of KRas-mutant NSCLC cells to cisplatin-induced apoptosis can be increased by overexpressing miR-202.

\section{Conclusion}

These data provide several pieces of evidence to demonstrate that the recovery of miR-202 expression enhances the effectiveness of platinum-based chemotherapy against KRas-mutant NSCLC by inhibiting the KRas/MAPK pathway. Considering these results, the combination of miR-202 with platinum-based chemotherapeutic drugs may represent an attractive treatment for patients with KRas-mutant NSCLC.

\section{Disclosure Statement}

The authors have no conflicts of interest.

\section{References}

1 Siegel R, Naishadham D, Jemal A: Cancer statistics, 2013. CA Cancer J Clin 2013;63:11-30.

2 Peng B, Wang YH, Liu YM and Ma LX: Prognostic signifcance of the neutrophil to lymphocyte ratio in patients with non-small cell lung cancer: a systemic review and meta-analysis. Int J Clin Exp Med 2015;8:3098-3106.

-3 Wang S, Liu F, Zhu J, Chen P, Liu H, Liu Q, Han J: DNA Repair Genes ERCC1 and BRCA1 Expression in NonSmall Cell Lung Cancer Chemotherapy Drug Resistance. Med Sci Monit 2016;22:1999-2005.

-4 Umihanic S, Umihanic S, Jamakosmanovic S, Brkic S, Osmic M, Dedic S, Ramic N: Glasgow prognostic score in patients receiving chemotherapy for non-small-cell lung cancer in stages IIIb and IV. Med Arch 2014;68:83-85.

5 Raghav KP, Gonzalez-Angulo AM, Blumenschein GR Jr: Role of HGF/MET axis in resistance of lung cancer to contemporary management. Transl Lung Cancer Res 2012;1:179-193.

-6 Tung MC, Lin PL, Wang YC, He TY, Lee MC, Yeh SD, Chen CY, Lee H: Mutant p53 confers chemoresistance in non-small cell lung cancer by upregulating Nrf2. Oncotarget 2015;6:41692-41705.

7 Uekita T, Fujii S, Miyazawa Y, Iwakawa R, Narisawa-Saito M, Nakashima K, Tsuta K, Tsuda H, Kiyono T, Yokota J, Sakai R: Oncogenic Ras/ERK signaling activates CDCP1 to promote tumor invasion and metastasis. Mol Cancer Res 2014;12:1449-1459.

8 Malumbres M, Barbacid M: RAS oncogenes: the first 30 years. Nat Rev Cancer 2003;3:459-465.

-9 Vasan N, Boyer JL, Herbst RS: A RAS renaissance: emerging targeted therapies for KRAS-mutated non-small cell lung cancer. Clin Cancer Res 2014;20:3921-3930.

10 Meng D, Yuan M, Li X, Chen L, Yang J, Zhao X, Ma W, Xin J: Prognostic value of K-RAS mutations in patients with non-small cell lung cancer: a systematic review with meta-analysis. Lung Cancer 2013;81:1-10.

11 Pylayeva-Gupta Y, Grabocka E, Bar-Sagi D: RAS oncogenes: weaving a tumorigenic web. Nat Rev Cancer 2011;11:761-774.

12 Park IH, Kim JY, Jung JI, Han JY: Lovastatin overcomes gefitinib resistance in human non-small cell lung cancer cells with K-Ras mutations. Invest New Drugs 2010;28:791-799.

13 Bartel DP: MicroRNAs: target recognition and regulatory functions. Cell 2009;136:215-233.

14 Iorio MV and Croce CM: microRNA involvement in human cancer. Carcinogenesis 2012;33:1126-1133.

15 Di Leva G, Garofalo M, Croce CM: MicroRNAs in cancer. Annu Rev Pathol 2014;9:287-314.

16 Han Z, Zhou X, Li S, Qin Y, Chen Y, Liu H: Inhibition of miR-23a increases the sensitivity of lung cancer stem cells to erlotinib through PTEN/PI3K/Akt pathway. Oncol Rep 2017;38:3064-3070. 


\section{Cellular Physiology Cell Physiol Biochem 2018;51:2160-2171 \begin{tabular}{ll|l} 
and & DO.1159/000495835 & $\begin{array}{l}\text { O } 2018 \text { The Author(s). Published by S. Karger AG, Basel } \\
\text { www.karger.com/cpb }\end{array}$
\end{tabular}}

Sun et al.: miR-202 Enhances Cisplatin on NSCLC

17 Jiang J, Feng X, Zhou W, Wu Y, Yang Y: MiR-128 reverses the gefitinib resistance of the lung cancer stem cells by inhibiting the c-met/PI3K/AKT pathway. Oncotarget 2016;7:73188-73199.

18 Yamamoto K, Seike M, Takeuchi S, Soeno C, Miyanaga A, Noro R, Minegishi Y, Kubota K, Gemma A: MiR379/411 cluster regulates IL-18 and contributes to drug resistance in malignant pleural mesothelioma. Oncol Rep 2014;32:2365-2372.

19 He H, Tian W, Chen H, Jiang K: MiR-944 functions as a novel oncogene and regulates the chemoresistance in breast cancer. Tumour Biol 2016;37:1599-1607.

-20 Livak KJ, Schmittgen TD: Analysis of relative gene expression data using real-time quantitative PCR and the 2(-Delta Delta C(T)) method. Methods 2001;25:402-408.

-21 Wang J, Tian X, Han R, Zhang X, Wang X, Shen H, Xue L. Liu Y, Yan X, Shen J, Mannoor K, Deepak J, Donahue JM, Stass SA, Xing L, Jiang F: Downregulation of miR-486-5p contributes to tumor progression and metastasis by targeting protumorigenic ARHGAP5 in lung cancer. Oncogene 2014;33:1181-1189.

-22 Shimamura T, Chen Z, Soucheray M, Carretero J, Kikuchi E, Tchaicha JH, Gao Y, Cheng KA, Cohoon TJ, Qi J, Akbay E, Kimmelman AC, Kung AL, Bradner JE, Wong KK: Efficacy of BET bromodomain inhibition in Krasmutant non-small cell lung cancer. Clin Cancer Res 2013;19:6183-6192.

23 Haydn JM, Hufnagel A, Grimm J, Maurus K, Schartl M, Meierjohann S: The MAPK pathway as an apoptosis enhancer in melanoma. Oncotarget 2014;5:5040-5053.

-24 Liu F, Zheng S, Liu T, Liu Q, Liang M, Li X, Sheyhidin I, Lu X, Liu W: MicroRNA-21 promotes the proliferation and inhibits apoptosis in Eca109 via activating ERK1/2/MAPK pathway. Mol Cell Biochem 2013;381:115125.

25 de Castria TB, da Silva EM, Gois AF, Riera R: Cisplatin versus carboplatin in combination with thirdgeneration drugs for advanced non-small cell lung cancer. Cochrane Database Syst Rev 2013;8:CD009256.

-26 Rotolo F, Dunant A, Le Chevalier T, Pignon JP, Arriagada R, IALT Collaborative Group: Adjuvant cisplatinbased chemotherapy in nonsmall-cell lung cancer: new insights into the effect on failure type via a multistate approach. Ann Oncol 2014;25:2162-2166.

-27 Tian Y, Zhang J, Yan S, Qiu L, Li Z: FATS expression is associated with cisplatin sensitivity in non small cell lung cancer. Lung Cancer 2012;76:416-422.

$28 \mathrm{Hu}$ J, Xu C, Cheng B, Jin L, Li J, Gong Y, Lin W, Pan Z, Pan C: Imperatorin acts as a cisplatin sensitizer via downregulating Mcl-1 expression in HCC chemotherapy. Tumour Biol 2016;37:331-339.

29 Li W, Zhang M, Xu L, Lin D, Cai S, Zou F: The apoptosis of non-small cell lung cancer induced by cisplatin through modulation of STIM1. Exp Toxicol Pathol 2013;65:1073-1081.

-30 Xu J, Yue CF, Zhou WH, Qian YM, Zhang Y, Wang SW, Liu AW, Liu Q: Aurora-A contributes to cisplatin resistance and lymphatic metastasis in non-small cell lung cancer and predicts poor prognosis. J Transl Med 2014;12:200.

31 Li N, Li X, Li S, Zhou S, Zhou Q: Cisplatin-induced downregulation of SOX1 increases drug resistance by activating autophagy in non-small cell lung cancer cell. Biochem Biophys Res Commun 2013;439:187-190.

-32 Yi Y, Li H, Lv Q Wu K, Zhang W, Zhang J, Zhu D, Liu Q, Zhang W: miR-202 inhibits the progression of human cervical cancer through inhibition of cyclin D1. Oncotarget 2016;7:72067-72075.

-33 Zhang Y, Zheng D, Xiong Y, Xue C, Chen G, Yan B, Ye Q: miR-202 suppresses cell proliferation in human hepatocellular carcinoma by downregulating LRP6 post-transcriptionally. FEBS Lett 2014;588:1913-1920.

34 Wang Q, Huang Z, Guo W, Ni S, Xiao X, Wang L, Huang D, Tan C, Xu Q, Zha R, Zhang J, Sheng W, He X, Du X: microRNA-202-3p inhibits cell proliferation by targeting ADP-ribosylation factor-like 5A in human colorectal carcinoma. Clin Cancer Res 2014;20:1146-1157.

-35 Shen X, Guo Y, Qi J, Shi W, Wu X, Ni H, Ju S: Study on the Association Between miRNA-202 Expression and Drug Sensitivity in Multiple Myeloma Cells. Pathol Oncol Res 2016;22:531-539.

36 Zhao Z, Lv B, Zhang L, Zhao N, Lv Y: miR-202 functions as a tumor suppressor in non-small cell lung cancer by targeting STAT3. Mol Med Rep 2017;16:2281-2289.

-37 Rejiba S, Wack S, Aprahamian M, Hajri A: K-ras oncogene silencing strategy reduces tumor growth and enhances gemcitabine chemotherapy efficacy for pancreatic cancer treatment. Cancer science. 2007;98:1128-1136.

-38 Kim WY, Prudkin L, Feng L, Kim ES, Hennessy B, Lee JS, Glisson B, Lippman SM, Wistuba II, Hong WK, Lee HY: Epidermal growth factor receptor and K-Ras mutations and resistance of lung cancer to insulin-like growth factor 1 receptor tyrosine kinase inhibitors. Cancer 2012;118:3993-4003. 
Cellular Physiology Cell Physiol Biochem 2018:51:2160-2171

\begin{tabular}{ll|l} 
and Biochemistry & $\begin{array}{l}\text { DOI: 10.1159/000495835 } \\
\text { Published onlIne: 6 December } 2018\end{array}$ & $\begin{array}{l}\text { @ } 2018 \text { The Author(s). Published by S. Karger AG, Basel } \\
\text { www.karger.com/cpb }\end{array}$ \\
\cline { 2 - 3 }
\end{tabular}

Sun et al.: miR-202 Enhances Cisplatin on NSCLC

-39 Vandamme D, Herrero A, Al-Mulla F and Kolch W: Regulation of the MAPK pathway by raf kinase inhibitory protein. Crit Rev Oncog 2014;19:405-415.

-40 Ercan D, Xu C, Yanagita M, Monast CS, Pratilas CA, Montero J, Butaney M, Shimamura T, Sholl L, Ivanova EV, Tadi M, Rogers A, Repellin C, Capelletti M, Maertens O, Goetz EM, Letai A, Garraway LA, Lazzara MJ, Rosen N et al.: Reactivation of ERK signaling causes resistance to EGFR kinase inhibitors. Cancer Discov 2012;2:934947.

41 Zhuo W, Zhang L, Zhu Y, Zhu B, Chen Z: Fisetin, a dietary bioflavonoid, reverses acquired Cisplatinresistance of lung adenocarcinoma cells through MAPK/Survivin/Caspase pathway. Am J Transl Res 2015;7:2045-2052. 